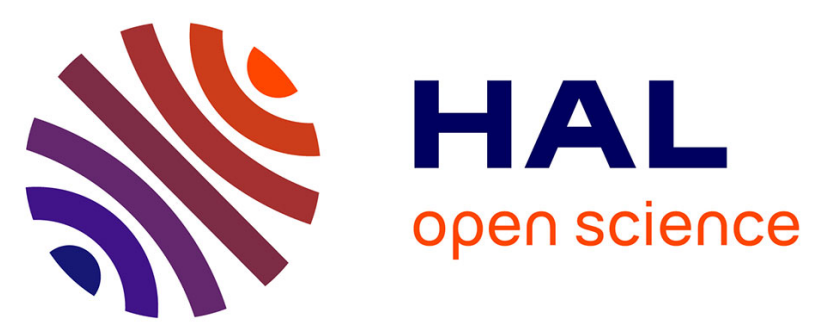

\title{
Adjoint-based numerical method using standard engineering software for the optimal placement of chlorine sensors in drinking water networks
}

Julien Waeytens, Imed Mahfoudhi, Mohamed-Amine Chabchoub, Patrice

Chatellier

\section{To cite this version:}

Julien Waeytens, Imed Mahfoudhi, Mohamed-Amine Chabchoub, Patrice Chatellier. Adjoint-based numerical method using standard engineering software for the optimal placement of chlorine sensors in drinking water networks. Environmental Modelling and Software, 2017, 92, pp.229-238. 10.1016/j.envsoft.2017.02.015 . hal-01488537

\section{HAL Id: hal-01488537 https://hal.science/hal-01488537}

Submitted on 13 Mar 2017

HAL is a multi-disciplinary open access archive for the deposit and dissemination of scientific research documents, whether they are published or not. The documents may come from teaching and research institutions in France or abroad, or from public or private research centers.
L'archive ouverte pluridisciplinaire HAL, est destinée au dépôt et à la diffusion de documents scientifiques de niveau recherche, publiés ou non, émanant des établissements d'enseignement et de recherche français ou étrangers, des laboratoires publics ou privés. 
$1 \quad$ Adjoint-based numerical method using standard engineering software for the optimal placement of chlorine sensors in drinking water networks

\author{
$4 \quad$ Julien Waeytens ${ }^{\mathrm{a}, *}$, Imed Mahfoudhi ${ }^{\mathrm{b}}$, Mohamed-Amine Chabchoub $^{\mathrm{a}}$, \\ $5 \quad$ Patrice Chatellier ${ }^{\mathrm{a}}$ \\ $6 \quad{ }^{a}$ Université Paris-Est, IFSTTAR, Marne-la-Vallée, 77447, France \\ $7 \quad{ }^{b}$ ENIM, University of Monastir, Monastir, 5000, Tunisie
}

\title{
8 Abstract
}

9 To obtain representative water quality simulations, unknown model param10 eters have to be updated by combining information from the water quality 11 model and the sensor outputs. An adjoint-based numerical method has 12 been developed to determine the optimal placement of chlorine sensors in 13 drinking water networks at a low computational cost. From a practical en14 gineering perspective, the proposed optimal placement corresponds to the set of sensors that minimizes the area in which the unknown model parameters cannot be identified. The numerical strategy is implemented in the

hydraulic software EPANET. Using the adjoint framework, we develop and apply an adaptive strategy in a French drinking water network that provides the optimal placement from 1 sensor to 6 sensors. We show that the highest reduction of the non-identifiable area is obtained at the first stages of the adaptive strategy. After 4 sensors, a plateau is reached.

${ }^{*}$ Corresponding author: E-mail: julien.waeytens@ifsttar.fr (J. Waeytens), Ph: +331 816684 53, Fax: +33 1816680 01, Postal address: Cit Descartes, 14-20 bd Newton, F-77447 Marne-la-Valle cedex 2, France 
27 Operating system: Windows network, adjoint method

\section{Software availability}

Name of software: EPANET

Programming language: $\mathrm{C} / \mathrm{C}++$

\section{Introduction}

Keywords: sensor placement, water quality simulations, drinking water

Availability: http://www.epa.gov/water-research/epanet

Documentation: http://www.epa.gov/water-research/epanet

User interface: Graphical user interface or Programmer's toolkit

License: Public domain software that may be freely copied and distributed

In drinking water networks, the chlorine concentration field is one of the main indicators of the water quality. Legislation dictates that a minimum level of chlorine at each point in the network has to be ensured. To overcome the lack of measurements in drinking water networks, hydraulic and water quality models are considered. In water network applications, the hydraulic state is generally computed using algebraic equations, i.e., flow continuity at the nodes and headloss in the pipes. Regarding the water quality models, one-dimensional (1D) advection-reaction equations are considered in pipes, and perfect and instantaneous mixing is assumed in pipe junctions. The decrease of the chlorine concentration due to bulk flow reactions and pipe wall reactions, e.g., reaction with the biofilm at the pipe wall, is modeled using a reaction term (Powell et al. (2000)). This term is characterized by the 
reaction order and the reaction coefficient. The software EPANET (Rossman and Boulos (1996); Rossman (2000)) is commonly used to simulate the hydraulics and the water quality states.

French water companies can observe a gap higher than $30 \%$ between the chlorine sensor outputs and the chlorine concentration obtained from a direct simulation of the water quality model. This gap may be due to uncertainties in the hydraulic state, particularly the water demands, and to the model parameters associated with the chlorine reactions. To represent the variability in water demands, stochastic models are typically considered. The calibration of these models can be achieved using direct measurements (Buchberger and Wells (1996); Bakker et al. (2013); Cominola et al. (2015)), i.e., monitoring of the user water consumption in residences, or indirect measurements (Kang and Lansey (2009); Alcocer-Yamanaka et al. (2012)), i.e., pressure and/or flow rate outputs into the drinking water network. To locate and quantify abnormal water demands due to leaks, inverse techniques based on pressure sensor outputs have been proposed in (Liggett and Chen (1994); Meseguer et al. (2014)). In this inverse problem, the goal is to determine the unknown model parameters by minimizing the gap between the sensor outputs and the simulation. Finally, flow sensor outputs may also be used in inverse techniques. Indeed, an inverse computational fluid dynamics technique has been developed in (Waeytens et al. (2015)) to identify the unknown boundary conditions of 2D incompressible Navier-Stokes equations and thus to obtain a high description in $2 \mathrm{D}$ of the flow profile in water networks. A detailed description of the flow in $2 \mathrm{D}$ or $3 \mathrm{D}$ can provide more representative chlorine simulations than using the mean flow velocity, particularly in the distribution mains of the drinking water networks where the flow can be laminar, thus inducing different chlorine propagation veloc- 
ities. Note that the measurement of chlorine or tracer concentrations can also provide information on the water demands (Jonkergouw et al. (2008); Al-Omari and Abdulla (2009)).

Regarding the water quality models, first-order reaction kinetics is commonly assumed for the free chlorine decay. Many articles address the identification of the reaction coefficient (Sharp et al. (1991); Rodriguez et al. (1997); Munavalli and Kumar (2005); Pasha and Lansey (2012)), but few aim to determine the reaction order and the reaction coefficient (Vasconcelos et al. (1997); Gancel (2006)). Because the reaction coefficient is associated with bulk flow reactions and pipe wall reactions, it is not uniform in the entire network. Nevertheless, to limit the number of unknowns to be determined, the reaction coefficient is considered to be piecewise constant on subsections of the water network. The choice of the domain decomposition is based on the age, the roughness, the pipe material, the pipe diameter and the flow rate.

Because drinking water networks are sparsely instrumented, the use of numerical tools can indicate to the water companies the coverage area ensured by the existing chlorine sensors and the optimal deployment of new chlorine sensors. A considerable amount of literature addresses the optimal sensor position for detecting a contaminant intrusion in drinking water networks. Three categories can be distinguished: the non-model-based methods using the topology of the water network, the methods based solely on hydraulic simulations (Lee and Deininger (1992); Kessler et al. (1998); Berry et al. (2005); Xu et al. (2008)) and the methods based on hydraulic and water quality simulations (Berry et al. (2006); Preis and Ostfeld (2008); Krause et al. (2008)). The majority of the methods formulate the optimal sensor placement as a multiobjective optimization. The goal is to minimize the 
non-coverage area, the number of sensors, the time to detection, and so forth.

In the present article, we propose a numerical strategy based on the adjoint framework to determine and quantify the non-coverage area for a given set of sensor placements. This practical information can be useful for water companies to determine the optimal placement of chlorine sensors for maximizing the coverage area for a given number of sensors. The method requires the resolution and the post-treatment of the solution of the adjoint problem, i.e., advection-reaction equations backward in time with virtual chlorine injection at the position of the sensors and a dynamic back flow. The adjoint framework is used in various applications. First, it provides at a low computational cost the functional gradient involved in inverse calculations to update the model parameters of the water flow (Liggett and Chen (1994); Waeytens et al. (2015)) and to reconstruct the concentration fields (Elbern et al. (2000); Waeytens et al. (2013, 2017)). Then, it is used in sensitivity analyses to study the influence of the physical model parameters on a quantity of interest (Andrews (2013); Kauker et al. (2016)). The adjoint framework is also considered for estimating the modeling or the discretization error on a quantity of interest (Becker and Rannacher (2001); Waeytens et al. (2012); Oden and Prudhomme (2002)). Note that the determination and quantification of the coverage area can also be obtained from (Xu et al. (2008)), which is based on the knowledge of the flow and graph theory. The main advantage of the proposed adjoint approach is that it uses standard hydraulic software such as EPANET. Moreover, the adjoint solution can also be used in an inverse advection-reaction procedure to identify the reaction coefficient.

The remainder of this article is organized as follows. Section 2 introduces the 
model updating technique for identifying the reaction coefficient of the water quality model and the definition of the adjoint problem. The adjoint-based numerical strategy for the optimal chlorine sensor placement is developed in Section 3. This strategy is applied to a French drinking water network in Section 4 before drawing concluding remarks and prospects in Section 5 .

\section{Modeling the water quality in drinking water networks and updating the kinetic reaction coefficient}

\subsection{Simulating the water quality in drinking water networks}

In drinking water networks, the chlorine concentration is the primary indicator of the water quality. The chlorine propagates in the network according to the flow induced by water demands, and the chlorine concentration decreases due to reactions occurring in the bulk or at the wall. Physical models can be employed to predict the propagation and the reaction of chlorine in drinking water networks. Generally, one-dimensional (1D) advection-reaction partial differential equations are considered in the pipes, and the mixing in the junctions is modeled using algebraic equations. The set of equations, detailed in (Rossman (2000)), for modeling the water quality in the drinking water network is called a "direct problem". It can be solved using standard engineering software such as EPANET (Rossman and Boulos (1996)). Let us define the simulated chlorine concentration in the water network as $\mathbf{C}$.

In practice, water companies may observe a gap higher than $30 \%$ between the simulated and measured chlorine concentrations. Hence, to obtain a representative simulation of the water quality, the model parameters, such as the kinetic reaction coefficient, have to be updated. The model updating 
strategy is described in the following sections.

\subsection{Cost functional used in model updating}

To obtain representative water quality simulations, one needs to update the unknown parameters of the model. Herein, we focus on determining the vector $\mathbf{k}=\left\{k_{1}, \ldots, k_{N}\right\}$ of reaction coefficients, where $N$ corresponds to the number of water pipes in the drinking water network. For this purpose, an inverse modeling technique can be employed. Let us choose a quadratic cost functional that quantifies the difference between the sensor outputs $C_{m}^{m e s}$ and the numerical solution $\mathbf{C}$ of the water quality model mentioned in the previous section. One seeks the vector $\mathbf{k}$ of reaction coefficients by solving the following optimization problem:

$\min _{\mathbf{k}} J(\mathbf{C}, \mathbf{k})=\frac{1}{2} \sum_{m=1}^{N} a_{m} \int_{0}^{T}\left(C_{m}\left(\mathbf{k} ; x=x_{m}, t\right)-C_{m}^{m e s}(t)\right)^{2} d t+\beta b \sum_{m=1}^{N}\left(k_{m}-k_{m}^{0}\right)^{2}$

where $\mathbf{C}=\left\{C_{1}(x, t), \ldots, C_{N}(x, t)\right\}$ is the vector of chlorine concentrations. The Boolean parameter $a_{m}$ is set to 1 (resp. 0) if the $m^{\text {th }}$ water pipe is equipped (resp. is not equipped) with a chlorine sensor recording the concentration level on the observation time interval $[0, T]$. The position of the $m^{\text {th }}$ sensor is denoted as $x_{m}$. Note that Eq. (1) is a constrained optimization problem. Indeed, the chlorine concentration field $\mathbf{C}$ has to satisfy the set of water quality equations mentioned in Section 2.1.

In general, the inverse problem is not well posed. First, in practice, to reduce the number of model parameters to be updated, the reaction coefficient is assumed to be the same on a group of water pipes that have the same characteristics, e.g., age, diameter and material (Fabrie et al. (2010)). Second, a Tikhonov regularization term such as the second term in Eq. (1) can be introduced in the cost functional. This term aims at improving the 
convexity of the functional. The parameter $b$ ensures the physical homogeneity of both terms, and $k_{m}^{0}$ corresponds to the initial guess of the $m^{\text {th }}$ reaction coefficient. $\beta$ corresponds to a normalized weighting coefficient.

The minimization of the cost functional (1) can be performed using a gradient-like approach. In the present article, note that the functional gradient is obtained at a low computational cost using the adjoint framework. The methodology to derive the adjoint equation and the gradient formula are presented in the next section.

\subsection{Derivation and physical meaning of adjoint equations}

The constrained minimization problem (1) can be rewritten as an unconstrained minimization problem by introducing the Lagrangian $\mathcal{L}(\mathbf{k}, \mathbf{C}, \mathbf{P})$ and the Lagrange multiplier $\mathbf{P}$. The stationarity of the Lagrangian according to the Lagrange multiplier $\mathbf{P}$ provides the equations of the direct problem mentioned in Section 2.1, whereas the equations of the adjoint problem are obtained from the stationarity of the Lagrangian according to $\mathbf{C}$.

Herein, the adjoint problem is quite similar to the direct problem. It is still an advection problem with a reaction term. In the adjoint problem, chlorine is virtually injected at the sensor location $x_{m}$. The temporal evolution of the chlorine injection is provided by the data misfit. In contrast to the direct problem, the adjoint problem has a final condition in time. Moreover, the flow is reversed in the adjoint problem, i.e., the velocity $\mathbf{v}$ is replaced by $(-\mathbf{v})$.

Physically, the adjoint state corresponds to a "sensitivity concentration". Considering a sensor at a given location in the drinking water network, the 
"sensitivity concentration" propagates from the sensor location toward the upstream flow direction with increasing reversed time. Hence, it shows that all of the sensitivity of the sensor measurement is located upstream of the sensor location. In other words, the sensor is not sensitive to locations downstream of the sensor, nor is it sensitive to chlorine concentrations that were present prior to the initial observation time.

The adjoint state can provide sensitivity information, but it can also be used to compute the functional gradient $\boldsymbol{\nabla} \boldsymbol{J}$, which is involved in model updating techniques, at a low computation cost. Indeed, $n+1$ transport reaction problems have to be solved in standard finite-difference techniques, whereas only two transport reaction problems are needed when using the adjoint framework to compute the $n$ components of the functional gradient. The functional gradient $\boldsymbol{\nabla} \boldsymbol{J}$ is obtained from the derivative of the Lagrangian according to the reaction coefficient $k_{m}$.

To provide a better understanding, the derivation of the adjoint equations and the functional gradient are illustrated on a divergent node of a water network in Appendix B.

\subsection{Practical technique to update the reaction coefficient of the water quality} model

Drinking water networks are not massively instrumented with chlorine sensors. As mentioned in Section 2.2, to limit the number of model parameters to be updated, the reaction coefficient is assumed to be uniform on subdomains of the water network that have the same characteristics. To update the vector $\mathbf{k}$ of reaction coefficients, one can follow the iterative 
strategy detailed below.

Direct problem: First, considering an initial guess $\mathbf{k}^{0}$ or the kinematic parameters obtained at the end of the previous iteration, the advectionreaction direct problem is solved, thereby providing the concentration field $\mathbf{C}$ in the water network.

Comparison of simulated chlorine and sensor outputs:. Knowing the simulated concentration field $\mathbf{C}$ in the entire water network, we compute the data misfit at each sensor location.

Adjoint problem: In a water network, the adjoint problem is defined as an advection-reaction problem backward in time considering a reversed flow. It corresponds to the retropropagation of chlorine virtually injected at the sensor location. The higher is the data misfit, the higher is the chlorine to be injected.

After changing the time variable $t$ to $\tau=T-t$, the final time condition begins as an initial condition. Hence, standard hydraulic and water quality software can be employed to solve the adjoint problem. By solving this problem, we obtain the adjoint state $\mathbf{P}$.

Functional gradient:. Let us consider the reaction coefficient $k_{p}$ modeling the chlorine reaction in a subdomain $\Omega_{p}$ of the water network. This subdomain is composed of $n_{p}$ water pipes. Thus, the formula of the derivative of the functional according to $k_{p}$ can be expressed as

$$
\frac{\partial J}{\partial k_{p}}=-\sum_{i=1}^{n_{p}} S_{i} \int_{0}^{T} \int_{0}^{L_{i}} C_{i} P_{i} d x d t+\beta b\left(k_{p}-k_{p}^{0}\right)
$$

In Eq. (2), the first term is associated with the sensitivity of the data misfit to the reaction coefficient $k_{p}$, and the second term is dedicated to the 
functional regularization.

All the components of the functional gradient $\boldsymbol{\nabla} \boldsymbol{J}$ are computed from Eq. (2).

Updating of the reaction coefficients: Using the functional gradient $\boldsymbol{\nabla} \boldsymbol{J}$ as the descent direction, we obtain the updated vector $\mathbf{k}_{\mathbf{n e w}}$ of reaction coefficients by

$$
\mathbf{k}_{\text {new }}=\mathbf{k}_{\text {old }}-\alpha \boldsymbol{\nabla} \boldsymbol{J}
$$

where $\alpha$ is the descent step. Several solutions are required to determine the descent step minimizing the cost functional $J$.

If the data misfit functional reaches the measurement error, the model updating process is stopped. Otherwise, the iterative process continues.

\section{Optimal chlorine sensor placement using the adjoint framework}

3.1. Theoretical foundations of the optimal chlorine sensor placement method

Proposition 3.1. Let us denote $\phi^{*}(x, t)$ as the modified adjoint solution.

If $\phi^{*}(x, t)=0$ in $\Omega_{p} \times[0, T]$, then the reaction coefficient $k(x)$ is not identifiable on the subdomain $\Omega_{p}$.

The modified adjoint solution $\phi^{*}(x, t)$ verifies the following transport equations in the pipes of the water networks

$-\frac{\partial \phi_{m}^{*}}{\partial t}-v_{m} \frac{\partial \phi_{m}^{*}}{\partial x}=a_{m} H(t) \delta\left(x-x_{m}\right), \quad$ in $\left[0, L_{m}\right] \times[0, T], m \in\{1, \ldots, N\}$

The boolean $a_{m}$ is equal to 1 (resp. 0) if the $m^{\text {th }}$ water pipe is equipped with a chlorine sensor (resp. is not equipped with a chlorine sensor). As with the adjoint problem, the pipe junctions are governed by the standard equations of convergent or divergent nodes depending on the direction of the flow 
$\left(-v_{m}\right)$. The flow $\left(-v_{m}\right)$ is considered stationary. Finally, in the modified adjoint problem, the final condition and the Dirichlet boundary conditions vanish.

Note that the modified adjoint problem resembles the adjoint problem. The two differences are as follows. First, no reaction term is involved in the modified adjoint problem. Second, in the adjoint problem, the amplitude of the injected chlorine at the sensor location is given by the data misfit, whereas a constant amplitude in time is considered in the modified adjoint problem.

The proof of Proposition 3.1 is given for a reduced water network in Appendix B. Although a reduced water network is considered to facilitate the notations, it includes key elements of a real water network, i.e., divergent and convergent nodes.

\subsection{Numerical method for optimal chlorine sensor placement}

For a given number $n_{s}$ of chlorine sensors, we seek the placement of chlorine sensors that minimizes the non-identifiable area associated with the reaction coefficient $k(x)$. For this purpose, we propose an adjoint-based numerical method, which is detailed below.

First, the hydraulic $v(x, t)$ has to be simulated in the drinking water network.

From the hydraulic, we deduce the reversed velocity field, i.e., $(-1) \times v(x, t)$. In agreement with the deployment constrains in the water network, a possible placement of $n_{s}$ chlorine sensor is considered. Then, the reversed velocity field is used to obtain the modified adjoint concentration $\phi^{*}(x, t)$. The modified adjoint problem, introduced in Proposition 3.1, corresponds to the retropropagation of chlorine virtually injected at the sensor locations 
with a constant unitary amplitude in time. In practice, after making the change of variable $\tau=T-t$, this problem can be solved using standard engineering software, e.g., EPANET. In Proposition 3.1, we show that information concerning the non-identifiable area associated with the reaction coefficient $k(x)$ can be provided by the modified adjoint concentration field $\phi^{*}(x, t)$. The subdomain $\Omega_{p}$ having a null modified adjoint concentration on the entire time interval $[0, T]$ corresponds to the non-identifiable area. To quantify and compare different sensor locations in view of minimizing the non-identifiable area, we introduce the dimensionless non-coverage indicator $\eta$, which is defined as

$$
\eta=\frac{\text { Total length of water pipes in the non-identifiable area }}{\text { Total length of water pipes in the drinking water network }}
$$

The proposed indicator is rapidly computable and readily usable. Thus, it meets the expectations of hydraulic engineers.

\section{Numerical results using the software EPANET}

Let us consider a part of the French drinking water network presented in Figure 1. The water network is composed of two tanks, 298 junctions and 318 pipes. The total water pipe length is approximately $15 \mathrm{~km}$. Due to technical and deployment constrains, chlorine sensors cannot be installed anywhere in the drinking water network. Hence, the water company Suez-Environnement has pre-selected 6 potential chlorine sensor positions, as presented in Figure 1. The chlorine sensors are useful for comparing the measurements and the water quality simulations. As we previously mentioned, more representative water quality simulations can be achieved by updating the unknown reaction coefficient $k(x)$. 
Our goal is to determine the best set of chlorine sensors that maximize the coverage area, i.e., minimizing the non-identifiable area associated with the reaction coefficient $k(x)$. In this section, we apply the proposed adjointbased numerical method. It has been implemented in the software EPANET.

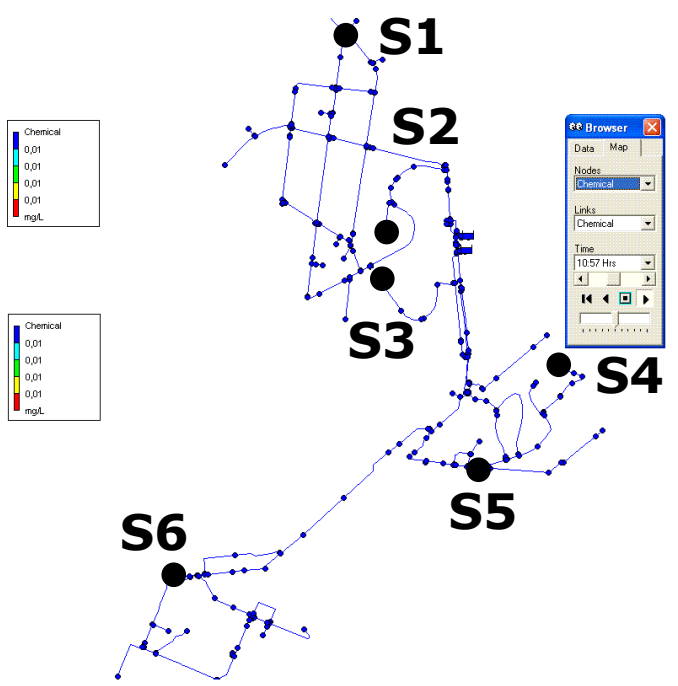

Figure 1: Geometry of a French drinking water network with 2 water towers - 6 possible locations of chlorine sensors

Following the methodology presented in the previous section, the first step consists of the hydraulic simulation in the water network using EPANET. As input for the hydraulic simulation, we use estimated varying consumer demands and the initial water level in water towers. The information associated with one day in August 2011 was provided by the water company Suez-Environnement. Then, we reverse the simulated flow, i.e., $-v(x, t)$, for simulating the modified adjoint problem $\phi^{*}(x, t)$. We recall that the modified adjoint problem is defined as the retropropagation of chlorine virtually 
injected at the sensor locations with a constant unitary amplitude in time.

As a first step, we consider the deployment of only one chlorine sensor in the water network. We test several locations of the sensor, and for each position from $S_{1}$ to $S_{6}$ (see Figure 1), we solve the associated modified adjoint problem. In Figure 2, we show the non-identifiable area when considering a sensor placed at $S_{1}$ or at $S_{6}$. The blue color (resp. the red color) denotes the area where the modified adjoint solution vanishes (resp. is not null) on the entire daily time interval. According to the theoretical results of the previous section, the blue color area is associated with the non-identifiable area. To quantify this area, the indicator $\eta$ defined in Eq. (5) is computed. The results are summarized in Table 1 . The non-identifiable area represents $87.5 \%$ of the water network for a sensor placed at $S_{1}$, whereas it represents $73.4 \%$ of the water network for $S_{6}$. Moreover, in Figure 2, we observe that a sensor placed at $S_{1}$ (resp. at $S_{6}$ ) is not able to provide information on chlorine reactions in the lower part of the network (resp. in the upper part of the network). When considering a unique sensor, note that the optimal sensor placement for minimizing the non-identifiable area corresponds to $S_{6}$.

To reduce the non-identifiable area, more chlorine sensors should be deployed in the water network. In the following, combinations of chlorine sensors are studied. In Figure 3, we can observe that when using the combination of the three chlorine sensors $S_{1}-S_{3}-S_{6}$, the non-identifiable area represents $54.4 \%$ of the drinking water network. Considering these 3 sensors rather than only sensor $S_{1}$ provides a $3 \mathrm{~km}$ reduction in the non-identifiable area. Nevertheless, increasing the number of sensors does not strongly reduce the non-identifiable area. Indeed, in Figures 3 and 4, we observe that from 3 to 5 chlorine sensors, the non-identifiable area indicator changes from 

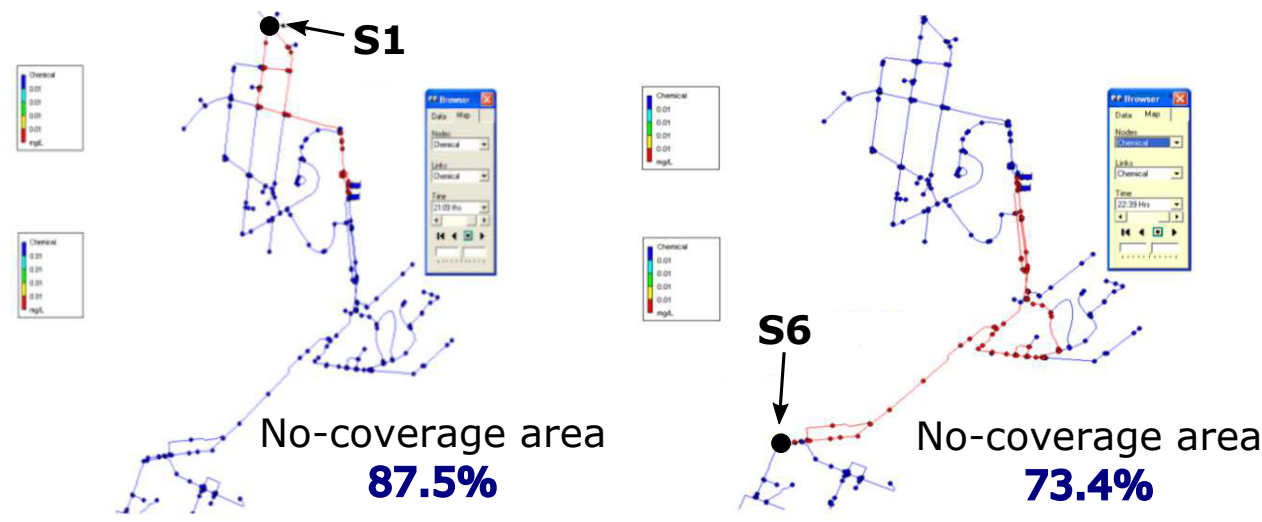

Figure 2: Sensor position $S_{1}: \eta=87.5 \%$ (left), Sensor position $S_{6}: \eta=73.4 \%$ (right)

\begin{tabular}{|l||l|}
\hline Sensor & Non-coverage indicator $\eta$ \\
\hline$S_{1}$ & $87.5 \%$ \\
\hline$S_{2}$ & $97.5 \%$ \\
\hline$S_{3}$ & $88.5 \%$ \\
\hline$S_{4}$ & $81.9 \%$ \\
\hline$S_{5}$ & $86.3 \%$ \\
\hline$S_{6}$ & $73.4 \%$ \\
\hline
\end{tabular}

Table 1: Non-coverage area of the drinking water network when considering a unique chlorine sensor

$54.4 \%$ to $48.1 \%$. No improvements are observed when adding a sixth sensor.

An adaptive strategy can be applied to obtain a desired threshold of non-identifiable area. The adaptive process starts by considering a unique chlorine sensor. Using the proposed adjoint-based technique, we retain the sensor placement $S_{I}^{\text {opt }}$ that has the lowest non-identifiable area indicator $\eta$. Then, to continue decreasing the non-identifiable area, an additional chlorine sensor is considered in the drinking water network. The indicator 


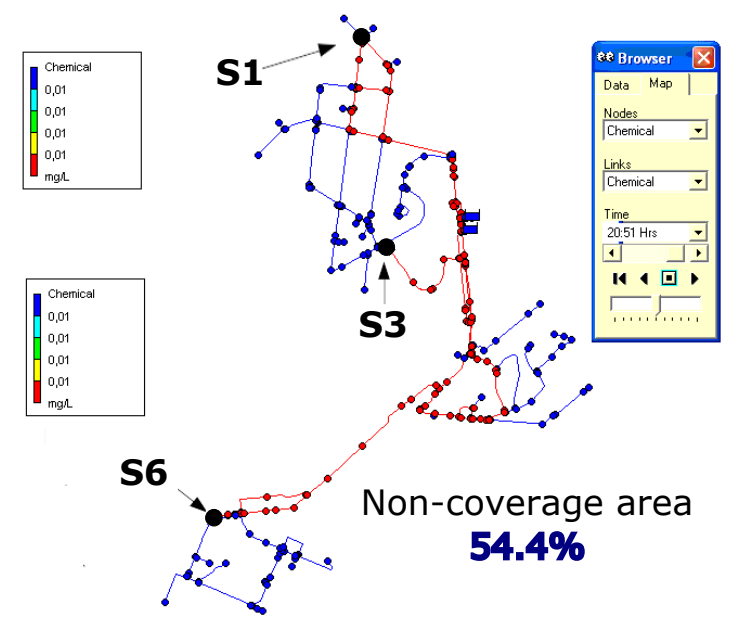

Figure 3: Combination of three sensors $S 6-S 1-S 3: \eta=54.4 \%$
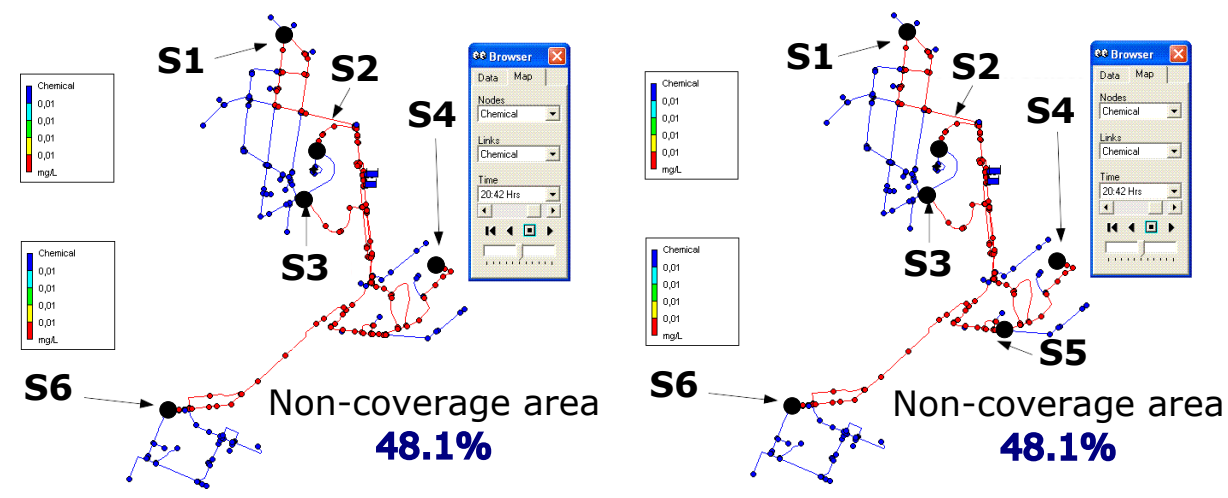

Figure 4: Combination of 5 sensors $S_{6}-S_{1}-S_{3}-S_{4}-S_{2}: \eta=48.1 \%$ (right), Combination of 6 sensors $S_{1}$ to $S_{6}: \eta=48.1 \%$ (left)

$369 \eta$ is computed for all combinations of two sensors, including the sensor $S_{I}^{\text {opt }}$ 370 determined at the previous stage. Hence, we obtain the optimal combination 371 of two sensors $\left(S_{I}^{o p t}, S_{I I}^{o p t}\right)$. The adaptive procedure continues until we reach 372 the maximum number of chlorine sensors affordable for the water network. 


\begin{tabular}{||l|l||l|l||l|l||}
\hline \hline 1 Sensor & $\begin{array}{l}\text { Non-coverage } \\
\text { indicator } \eta\end{array}$ & 2 Sensors & $\begin{array}{l}\text { Non-coverage } \\
\text { indicator } \eta\end{array}$ & 3 Sensors & $\begin{array}{l}\text { Non-coverage } \\
\text { indicator } \eta\end{array}$ \\
\hline \hline$S_{1}$ & $87.5 \%$ & $\mathbf{S}_{\mathbf{6}}, \mathbf{S}_{\mathbf{1}}$ & $\mathbf{6 1 . 7} \%$ & $S_{6}, S_{1}, S_{2}$ & $59.2 \%$ \\
$S_{2}$ & $97.5 \%$ & $S_{6}, S_{2}$ & $70.9 \%$ & $\mathbf{S}_{\mathbf{6}}, \mathbf{S}_{\mathbf{1}}, \mathbf{S}_{\mathbf{3}}$ & $\mathbf{5 4 . 4} \%$ \\
$S_{3}$ & $88.5 \%$ & $S_{6}, S_{3}$ & $63.5 \%$ & $S_{6}, S_{1}, S_{4}$ & $56.1 \%$ \\
$S_{4}$ & $81.9 \%$ & $S_{6}, S_{4}$ & $67.8 \%$ & $S_{6}, S_{1}, S_{5}$ & $61.8 \%$ \\
$S_{5}$ & $86.3 \%$ & $S_{6}, S_{5}$ & $72.1 \%$ & & \\
$\mathbf{S}_{\mathbf{6}}$ & $\mathbf{7 3 . 4 \%}$ & & & & \\
\hline \hline
\end{tabular}

Table 2: Non-coverage area of the drinking water network when considering 1, 2 or 3 chlorine sensors - optimal combinations of sensors are noted in bold

\begin{tabular}{||l|l||l|l||l||}
\hline \hline 4 Sensors & $\begin{array}{l}\text { Non-coverage } \\
\text { indicator } \eta\end{array}$ & 5 Sensors & $\begin{array}{l}\text { Non-coverage } \\
\text { indicator } \eta\end{array}$ & $\begin{array}{l}\text { Non-coverage } \\
\text { indicator } \eta\end{array}$ \\
\hline \hline$S_{6}, S_{1}, S_{3}, S_{2}$ & $53.7 \%$ & $\mathbf{S}_{\mathbf{6}}, \mathbf{S}_{1}, \mathbf{S}_{\mathbf{3}}, \mathbf{S}_{\mathbf{4}}, \mathbf{S}_{\mathbf{2}}$ & $\mathbf{4 8 . 1} \%$ & $48.8 \%$ \\
$\mathbf{S}_{\mathbf{6}}, \mathbf{S}_{\mathbf{1}}, \mathbf{S}_{\mathbf{3}}, \mathbf{S}_{\mathbf{4}}$ & $\mathbf{4 8 . 8} \%$ & $S_{6}, S_{1}, S_{3}, S_{4}, S_{5}$ & $48.8 \%$ & $\mathbf{S}_{\mathbf{6}}, \mathbf{S}_{\mathbf{1}}, \mathbf{S}_{\mathbf{3}}, \mathbf{S}_{\mathbf{4}}, \mathbf{S}_{\mathbf{2}}, \mathbf{S}_{\mathbf{5}}$ \\
$S_{6}, S_{1}, S_{3}, S_{5}$ & $54.4 \%$ & & & \\
\hline \hline
\end{tabular}

Table 3: Non-coverage area of the drinking water network when considering 4, 5 or 6 chlorine sensors - optimal combinations of sensors are noted in bold

\section{Conclusions}

To obtain representative water quality simulations in drinking water networks, the unknown model parameters, such as the reaction coefficient, 


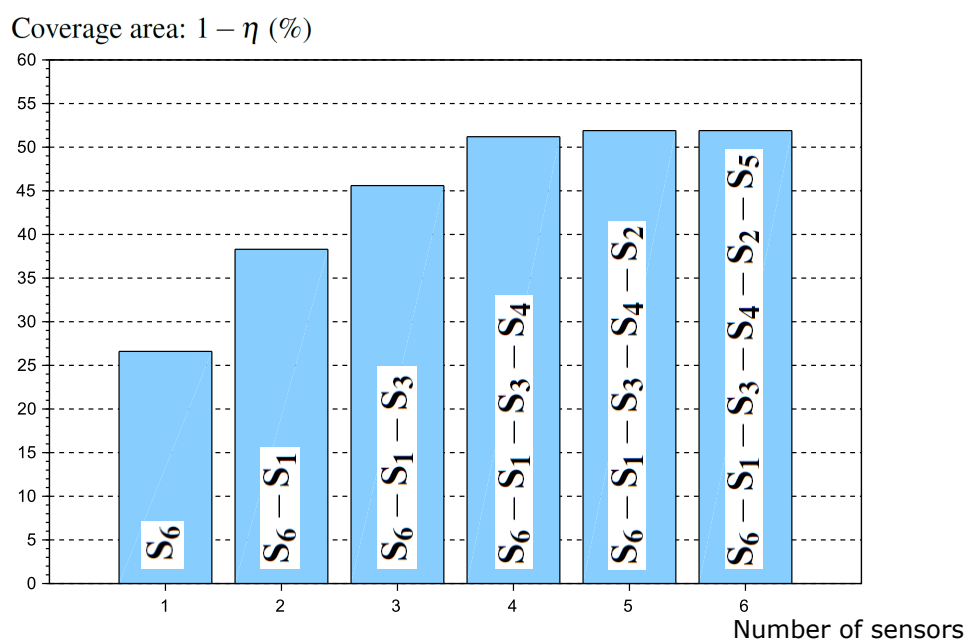

Figure 5: Synthesis of optimal sensor placement to maximize the coverage area

should be updated using chlorine sensor outputs. In the present article, an adjoint-based numerical method dedicated to drinking water networks has been developed to optimally deploy chlorine sensors in view of minimizing the non-identifiable area associated with the reaction coefficient. The computation of the one-dimensional adjoint advection solution using the standard engineering software EPANET allows us to localize and quantify the non-coverage area for a given set of sensors. On a French drinking water network, we applied an adaptive strategy starting from the optimal placement of 1 sensor to 6 sensors. We showed that the highest reduction in the non-identifiable area is obtained at the first stages of the adaptive strategy. After 4 sensors, a plateau is reached. In the model updating process of the reaction coefficient, the computed adjoint solution can also be used to obtain the functional gradient at a lower computational cost than straightforward difference techniques. Herein, we focus on the optimal sensor placement that 
minimizes the non-coverage area. The non-coverage area indicator computed from the modified adjoint solution can be used in a more general optimal sensor placement strategy considering the minimization of a multiobjective function. Finally, the proposed method can be extended for the detection of species intrusion in drinking water networks. The modified adjoint solution can highlight the area where species intrusion may not be detected.

\section{Acknowledgments}

We wish to thank the water company Suez-Environnement for providing geometric and hydraulic details of the French water networks studied in the present article. This research was supported by the French Inter-Ministry Fund (FUI) within the project "Micad'Eau", which involves several partners: Advitam, Ondeo Systems (Suez Environnement), EFS, A3IP, ESIEE Paris, and IFSTTAR.

\section{Appendix A. Illustration of adjoint framework on a divergent node of a water network and computation of the func- tional gradient}

The advection reaction within a divergent node (see Figure A.6) is represented by the following equations:

$$
\begin{array}{ll}
\frac{\partial C_{m}}{\partial t}+v_{m} \frac{\partial C_{m}}{\partial x}+k_{m} C_{m}=0 & \text { in }\left[0, L_{m}\right] \times[0, T], m \in\{1,2,3\} \\
C_{1}(x=0, t)=\chi_{1}(t) & \text { in }[0, T] \\
C_{2}(x=0, t)=C_{1}\left(x=L_{1}, t\right) & \text { in }[0, T] \\
C_{3}(x=0, t)=C_{1}\left(x=L_{1}, t\right) & \text { in }[0, T] \\
C_{m}(x, t=0)=c_{m}^{0}(x) & \text { in }\left[0, L_{m}\right], m \in\{1,2,3\}
\end{array}
$$


where $m$ denotes the pipe number, $L_{m}$ is the length of pipe $m, C_{m}$ is the chlorine concentration in water pipe $m$ as a function of distance $x$ and time $t, v_{m}$ is the flow velocity, and $k_{m}\left(\right.$ resp. $\left.\alpha_{m}\right)$ represents the reaction coefficient (resp. the reaction order). As usual in drinking water networks, a first-order reaction is considered to model the chlorine reaction. In the following, we take $\alpha_{m}=1$. The concentration boundary condition is $\chi_{1}(t)$, and $c_{m}^{0}(x)$ denotes the initial chlorine concentration in water pipe $m$.

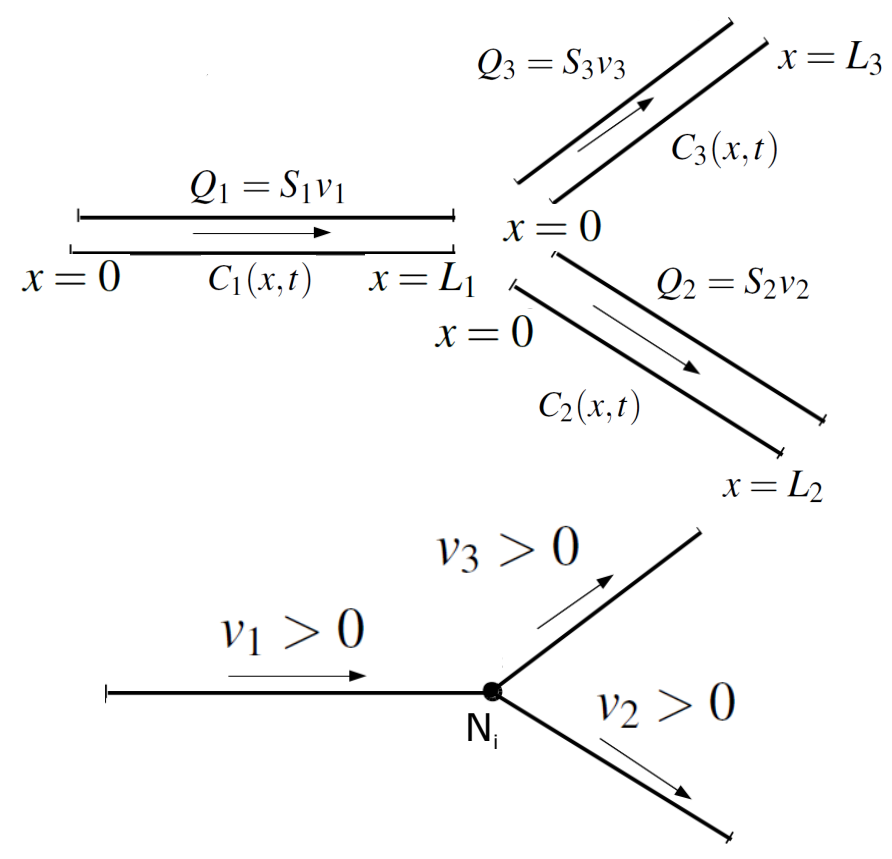

Figure A.6: Notations for advection reaction through divergent node - the flow velocity $v_{m}$ in pipe $m$ is considered positive when it passes from the starting node $(x=0)$ to the ending node $\left(x=L_{m}\right)$ - herein, $v_{1}>0, v_{2}>0$ and $v_{3}>0$

To derive the adjoint equations and the gradient formula, we introduce 
424

$$
\begin{array}{r}
\mathcal{L}(\mathbf{k}, \mathbf{C}, \mathbf{P}, \boldsymbol{\lambda}, \boldsymbol{\mu})=J(\mathbf{C}, \mathbf{k})-\sum_{m=1}^{3} S_{m} \int_{0}^{T} \int_{0}^{L_{m}}\left(\frac{\partial C_{m}}{\partial t}+v_{m} \frac{\partial C_{m}}{\partial x}+k_{m} C_{m}\right) P_{m}(x, t) d x d t \\
-S_{1} \int_{0}^{T}\left(C_{1}(x=0, t)-\chi_{1}(t)\right) \lambda_{1} d t-\sum_{m=2}^{3} S_{m} \int_{0}^{T}\left(C_{m}(x=0, t)-C_{1}\left(x=L_{1}, t\right)\right) \lambda_{m} d t \\
-\sum_{m=1}^{3} S_{m} \int_{0}^{L_{m}}\left(C_{m}(x, t=0)-c_{m}^{0}(x)\right) \mu_{m} d x
\end{array}
$$

425 and the cost functional is defined as

$$
J(\mathbf{C}, \mathbf{k})=\frac{1}{2} \sum_{m=1}^{3} a_{m} \int_{0}^{T}\left(C_{m}\left(\mathbf{k} ; x_{m}\right)-C_{m}^{m e s}\right)^{2} d t+\beta b \sum_{m=1}^{3}\left(k_{m}-k_{m}^{0}\right)^{2}
$$

${ }_{427} \quad$ where $\mathbf{k}=\left(\begin{array}{c}k_{1} \\ k_{2} \\ k_{3}\end{array}\right), \mathbf{C}=\left(\begin{array}{c}C_{1} \\ C_{2} \\ C_{3}\end{array}\right), \mathbf{P}=\left(\begin{array}{c}p_{1} \\ p_{2} \\ p_{3}\end{array}\right), \lambda=\left(\begin{array}{c}\lambda_{1} \\ \lambda_{2} \\ \lambda_{3}\end{array}\right)$ and $\mu=\left(\begin{array}{l}\mu_{1} \\ \mu_{2} \\ \mu_{3}\end{array}\right)$ ${ }_{428}$ and $S_{m}$ denotes the cross-sectional area of the $m^{\text {th }}$ water pipe.

429

433

434
As mentioned in Section 2.3, by writing the stationarity of the Lagrangian according to the Lagrange multipliers $\mathbf{P}, \boldsymbol{\lambda}$ and $\boldsymbol{\mu}$, we obtain the equations of the direct problem (A.1).

The equations of the adjoint problem are obtained from the stationarity 
435

$$
\begin{array}{rr}
\frac{\partial \mathcal{L}}{\partial C_{1}} \delta C_{1}=0 \Rightarrow & a_{1} \int_{0}^{T} \int_{0}^{L_{1}}\left(C_{1}\left(x_{1}\right)-C_{1}^{m e s}\right) \delta\left(x-x_{1}\right) \delta C_{1} d x d t \\
& -S_{1} \int_{0}^{T} \int_{0}^{L_{1}}\left(\frac{\partial \delta C_{1}}{\partial t}+v_{1} \frac{\partial \delta C_{1}}{\partial x}+k_{1} \delta C_{1}\right) P_{1}(x, t) d x d t-S_{1} \int_{0}^{T} \delta C_{1}(x=0, t) \lambda_{1} d t \\
& +\sum_{m=2}^{3} S_{m} \int_{0}^{T} \delta C_{1}\left(x=L_{1}, t\right) \lambda_{m} d t-S_{1} \int_{0}^{L_{1}} \delta C_{1}(x, t=0) \mu_{1} d x=0, \forall \delta C_{1} \\
\frac{\partial \mathcal{L}}{\partial C_{m}} \delta C_{m}=0 \Rightarrow \begin{array}{r}
a_{m} \int_{0}^{T} \int_{0}^{L_{m}}\left(C_{m}\left(x_{m}\right)-C_{m}^{m e s}\right) \delta\left(x-x_{m}\right) \delta C_{m} d x d t \\
\end{array} & -S_{m} \int_{0}^{T} \int_{0}^{L_{m}}\left(\frac{\partial \delta C_{m}}{\partial t}+v_{m} \frac{\partial \delta C_{m}}{\partial x}+k_{m} \delta C_{m}\right) P_{m}(x, t) d x d t \\
& -S_{m} \int_{0}^{T} \delta C_{m}(x=0, t) \lambda_{m} d t-S_{m} \int_{0}^{L_{m}} \delta C_{m}(x, t=0) \mu_{m} d x=0, \forall \delta C_{m}, m=2,3
\end{array}
$$

436

437 After integrating by parts, one obtains

$$
\begin{array}{r}
S_{m} \int_{0}^{T} \int_{0}^{L_{m}} \frac{\partial \delta C_{m}}{\partial t} P_{m}(x, t) d x d t= \\
S_{m} \int_{0}^{L_{m}} P_{m}(x, t=T) \delta C_{m}(x, t=T) d x \\
-S_{m} \int_{0}^{L_{m}} P_{m}(x, t=0) \delta C_{m}(x, t=0) d x \\
-S_{m} \int_{0}^{T} \int_{0}^{L_{m}} \frac{\partial P_{m}}{\partial t} \delta C_{m}(x, t) d x d t \\
S_{m} \int_{0}^{T} \int_{0}^{L_{m}} v_{m} \frac{\partial \delta C_{m}}{\partial x} P_{m}(x, t) d x d t=S_{m} v_{m} \int_{0}^{T} P_{m}\left(x=L_{m}, t\right) \delta C_{m}\left(x=L_{m}, t\right) d t \\
-S_{m} v_{m} \int_{0}^{T} P_{m}(x=0, t) \delta C_{m}(x=0, t) d t \\
-S_{m} \int_{0}^{T} \int_{0}^{L_{m}} v_{m} \frac{\partial P_{m}}{\partial x} \delta C_{m}(x, t) d x d t
\end{array}
$$

From Eqs. (A.4) and (A.5), we deduce the system of equations associated 439 with the stationarity of the Lagrangian according to the concentration field 


$$
\begin{aligned}
& -\frac{\partial P_{m}}{\partial t}-v_{m} \frac{\partial P_{m}}{\partial x}+k_{m} P_{m}=\frac{a_{m}}{S_{m}}\left(C\left(x_{m}, t\right)-C_{m}^{m e s}(t)\right) \delta\left(x-x_{m}\right), \text { in }\left[0, L_{m}\right] \times[0, T], m \in\{1,2,3\} \\
& P_{m}(x, t=T)=0, \quad \text { in }\left[0, L_{m}\right], m \in\{1,2,3\} \\
& S_{1} v_{1} P_{1}(x=L, t)=S_{2} \lambda_{2}+S_{3} \lambda_{3}, \quad \text { in }[0, T] \\
& P_{m}\left(x=L_{m}, t\right)=0, \quad \text { in }\left[0, L_{m}\right], m \in\{2,3\} \\
& \mu_{m}=P_{m}(x, t=0), \quad \text { in }\left[0, L_{m}\right], m \in\{1,2,3\} \\
& \lambda_{m}=v_{m} P_{m}(x=0, t), \quad \text { in }[0, T], m \in\{1,2,3\}
\end{aligned}
$$

441

In Eq. (A.6), note that the Lagrange multiplier $P_{m}$ corresponds to the adjoint state. Thus, the equations of the adjoint are given by

$$
\begin{aligned}
& -\frac{\partial P_{m}}{\partial t}-v_{m} \frac{\partial P_{m}}{\partial x}+k_{m} P_{m}=\frac{a_{m}}{S_{m}}\left(C\left(x_{m}, t\right)-C_{m}^{m e s}(t)\right) \delta\left(x-x_{m}\right), \quad \text { in }\left[0, L_{m}\right] \times[0, T], m \in\{1,2,3\} \\
& P_{m}(x, t=T)=0, \quad \text { in }\left[0, L_{m}\right], m \in\{1,2,3\} \\
& S_{1} v_{1} P_{1}\left(x=L_{1}, t\right)=S_{2} v_{2} P_{2}(x=0, t)+S_{3} v_{3} P_{3}(x=0, t), \quad \text { in }[0, T] \\
& P_{m}(x=L, t)=0, \quad \text { in }\left[0, L_{m}\right], m \in\{2,3\}
\end{aligned}
$$

From Eq. (A.7), we observe that the adjoint problem of an advectionreaction problem through a divergent node corresponds to an advectionreaction problem through a convergent node. In contrast to the direct problem, the adjoint problem is backward in time, i.e., it has a final condition, and the flow is reversed in the adjoint problem, i.e., the velocity $v_{m}$ is replaced by $\left(-v_{m}\right)$. The source term at the first line in Eq. (A.7) indicates that chlorine is virtually injected at sensor location $x_{m}$, and its amplitude is given by the data misfit. Hence, the chlorine retropropagates from the sensor locations.

In the same way, when considering an advection-reaction direct problem through a convergent node, it can be shown that its adjoint problem begins 
as an advection-reaction problem backward in time through a divergent node.

The functional gradient can be expressed using the adjoint state. It allows computation of the functional gradient at a lower computational cost than standard finite difference schemes. The analytical formula of the functional gradient can be obtained from the derivative of the Lagrangian according to the reaction coefficient $k_{m}$.

$$
\boldsymbol{\nabla} \boldsymbol{J}=\left(\begin{array}{c}
\frac{\partial J}{\partial k_{1}} \\
\frac{\partial J}{\partial k_{2}} \\
\frac{\partial J}{\partial k_{3}}
\end{array}\right)=\left(\begin{array}{l}
\frac{\partial \mathcal{L}}{\partial k_{1}} \\
\frac{\partial \mathcal{L}}{\partial k_{2}} \\
\frac{\partial \mathcal{L}}{\partial k_{3}}
\end{array}\right)=\left(\begin{array}{l}
-S_{1} \int_{0}^{T} \int_{0}^{L_{m}} C_{1} P_{1} d x d t+\beta b\left(k_{1}-k_{1}^{0}\right) \\
-S_{2} \int_{0}^{T} \int_{0}^{L_{m}} C_{2} P_{2} d x d t+\beta b\left(k_{2}-k_{2}^{0}\right) \\
-S_{3} \int_{0}^{T} \int_{0}^{L_{m}} C_{3} P_{3} d x d t+\beta b\left(k_{3}-k_{3}^{0}\right)
\end{array}\right)
$$

\section{Appendix B. Proof of Proposition 4.1 on a reduced water network}

In the interests of simplifying notations, let us consider the reduced water network presented in Figure B.7. It is composed of the main elements of a water network, i.e., water pipes, convergent nodes and divergent nodes. Following the methodology presented in Section 2.3 and illustrated in Appendix A, we can show that the adjoint problem associated with the reduced water network corresponds to

$$
\begin{aligned}
& -\frac{\partial P_{m}}{\partial t}-v_{m} \frac{\partial P_{m}}{\partial x}+k_{m} P_{m}=a_{m} f_{m}(x, t), \quad \text { in }\left[0, L_{m}\right] \times[0, T], m \in\{1,2,3,4\} \\
& P_{m}(x, t=T)=0, \quad \text { in }\left[0, L_{m}\right], m \in\{1,2,3,4\} \\
& P_{4}\left(x=L_{4}, t\right)=0, \quad \text { in }[0, T] \\
& P_{m}\left(x=L_{m}, t\right)=P_{4}(x=0, t), \quad \text { in }[0, T], m \in\{2,3\} \\
& S_{1} v_{1} P_{1}\left(x=L_{1}, t\right)=S_{2} v_{2} P_{2}(x=0, t)+S_{3} v_{3} P_{3}(x=0, t), \quad \text { in }[0, T]
\end{aligned}
$$



sensor location. It is defined by

$$
f_{m}(x, t)=\left(C\left(x_{m}, t\right)-C_{m}^{m e s}(t)\right) \delta\left(x-x_{m}\right) / S_{m} .
$$

a)

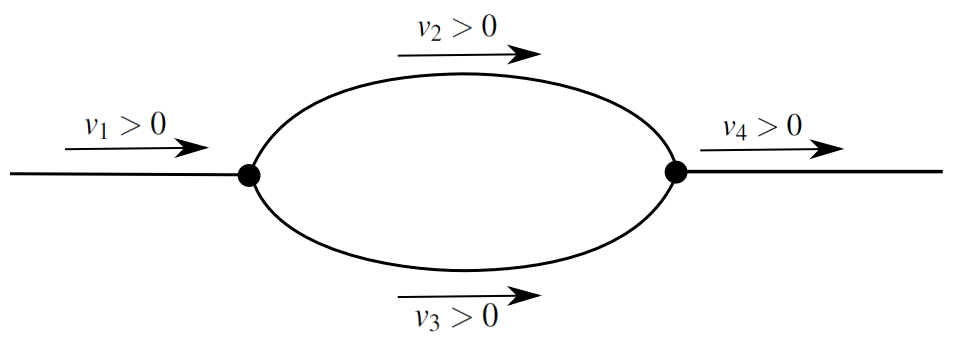

b)

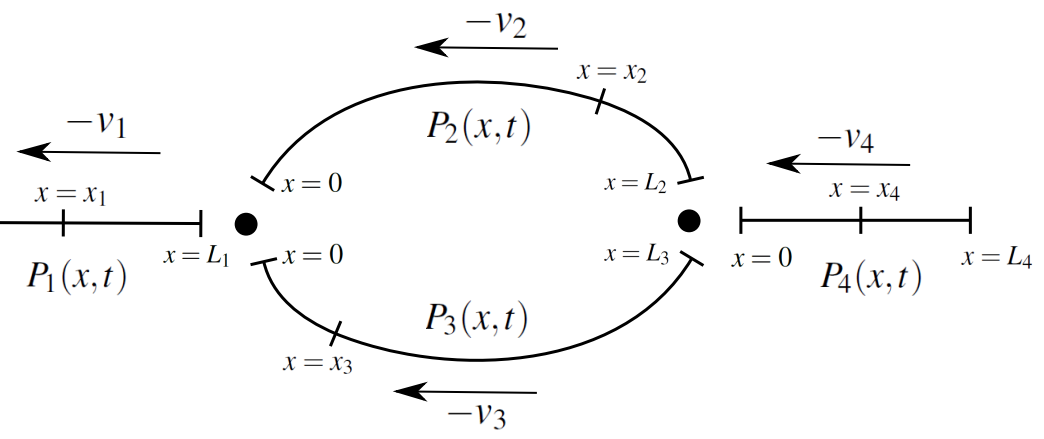

Figure B.7: Reduced water network: a) Flow direction b) Notations associated with the adjoint problem; in the advection-reaction adjoint problem, the flow is reversed; $\left\{x_{1}, x_{2}, x_{3}, x_{4}\right\}$ denote the potential locations of chlorine sensors

When considering no reaction term in (B.1), we obtain the no-reaction 


$$
\begin{aligned}
& -\frac{\partial \phi_{m}}{\partial t}-v_{m} \frac{\partial \phi_{m}}{\partial x}=a_{m} f_{m}(x, t), \quad \text { in }\left[0, L_{m}\right] \times[0, T], m \in\{1,2,3,4\} \\
& \phi_{m}(x, t=T)=0, \quad \text { in }\left[0, L_{m}\right], m \in\{1,2,3,4\} \\
& \phi_{4}\left(x=L_{4}, t\right)=0, \quad \text { in }[0, T] \\
& \phi_{m}\left(x=L_{m}, t\right)=\phi_{4}(x=0, t), \quad \text { in }[0, T], m \in\{2,3\} \\
& S_{1} v_{1} \phi_{1}\left(x=L_{1}, t\right)=S_{2} v_{2} \phi_{2}(x=0, t)+S_{3} v_{3} \phi_{3}(x=0, t), \quad \text { in }[0, T]
\end{aligned}
$$

$$
\begin{aligned}
& -\frac{\partial \phi_{m, i}^{*}}{\partial t}-v_{m} \frac{\partial \phi_{m, i}^{*}}{\partial x}=H(t) \delta\left(x-x_{i}\right), \quad \text { in }\left[0, L_{m}\right] \times[0, T], m=i \\
& -\frac{\partial \phi_{m, i}^{*}}{\partial t}-v_{m} \frac{\partial \phi_{m, i}^{*}}{\partial x}=0, \quad \text { in }\left[0, L_{m}\right] \times[0, T], m \neq i \\
& \phi_{m, i}^{*}(x, t=T)=0, \quad \text { in }\left[0, L_{m}\right], m \in\{1,2,3,4\} \\
& \phi_{4, i}^{*}\left(x=L_{4}, t\right)=0, \quad \text { in }\left[0, L_{4}\right] \\
& \phi_{m, i}^{*}\left(x=L_{m}, t\right)=\phi_{4, i}^{*}(x=0, t), \quad \text { in }\left[0, L_{m}\right], m \in\{2,3\} \\
& S_{1} v_{1} \phi_{1, i}^{*}\left(x=L_{1}, t\right)=S_{2} v_{2} \phi_{2, i}^{*}(x=0, t)+S_{3} v_{3} \phi_{3, i}^{*}(x=0, t), \quad \text { in }[0, T]
\end{aligned}
$$

$$
\phi_{m}^{*}(x, t)=\sum_{i=1}^{N} a_{i} \phi_{m, i}^{*}(x, t), \quad \text { in }\left[0, L_{m}\right] \times[0, T], m \in\{1,2,3,4\}
$$

482 On the one hand, the solution $\phi_{m}(x, t)$ of the no-reaction adjoint problem ${ }_{483}$ (B.3) can be obtained from the modified adjoint solution $\phi_{m, i}^{*}$ (also called 
"Green function"). Indeed, noting that

$$
f_{m}(t)=\left[f_{m} * \delta\right](t)=\left[f_{m}^{\prime} * H\right](t)
$$

485

486

$$
\phi_{m}(x, t)=\sum_{i=1}^{N} a_{i}\left[f_{i}^{\prime} * \phi_{m, i}^{*}\right](x, t), m \in\{1,2,3,4\}
$$

487

488

489

$\phi_{m}^{*}(x, t)=0$ in $\left[0, L_{m}\right] \times[0, T] \Leftrightarrow \phi_{m, i}^{*}(x, t)=0$ in $\left[0, L_{m}\right] \times[0, T] \Rightarrow \phi_{m}(x, t)=0$ in $\left[0, L_{m}\right] \times[0, T]$

490

491 On the other hand, subtracting Eq. (B.1) from (B.3) and noting $\delta P_{m}(x, t)=$ 492

$P_{m}(x, t)-\phi_{m}(x, t)$, one has

$$
\begin{aligned}
& -\frac{\partial \delta P_{m}}{\partial t}-v_{m} \frac{\partial \delta P_{m}}{\partial x}+k_{m} \delta P_{m}=-k_{m} \phi_{m}, \quad \text { in }\left[0, L_{m}\right] \times[0, T], m \in\{1,2,3,4\} \\
& \delta P_{m}(x, t=T)=0, \quad \text { in }\left[0, L_{m}\right], m \in\{1,2,3,4\} \\
& \delta P_{4}\left(x=L_{4}, t\right)=0, \quad \text { in }[0, T] \\
& \delta P_{m}\left(x=L_{m}, t\right)=\delta P_{4}(x=0, t), \quad \text { in }[0, T], m \in\{2,3\} \\
& S_{1} v_{1} \delta P_{1}\left(x=L_{1}, t\right)=S_{2} v_{2} \delta P_{2}(x=0, t)+S_{3} v_{3} \delta P_{3}(x=0, t), \quad \text { in }[0, T]
\end{aligned}
$$

493 From Eq. (B.9), we deduce that

$$
\left\{\begin{array}{lc}
\phi_{m}(x, t)=0, & \text { in }\left[0, L_{m}\right] \times[0, T] \\
\delta P_{m}\left(x=L_{m}, t\right)=0, & \text { in }[0, T] \\
\delta P_{m}(x, t=T)=0, & \text { in }\left[0, L_{m}\right]
\end{array}\right.
$$


494

$$
\delta P_{m}(x, t)=0, \quad \text { in }\left[0, L_{m}\right] \times[0, T] \Rightarrow P_{m}(x, t)=0, \text { in }\left[0, L_{m}\right] \times[0, T]
$$

495

496

497

498

As the gradient $\nabla J$ can be expressed using the adjoint solution $P_{m}$

$$
\frac{\partial J}{\partial k_{m}} \delta k_{m}=-S_{m} \int_{0}^{T} \int_{0}^{L_{m}} C_{m} P_{m} \delta k_{m} d x d t+\beta b\left(k_{m}-k_{m}^{0}\right) \delta k_{m}
$$

it can be noted that a null adjoint solution $P_{m}(x, t)$ in the pipe $m$ on the entire time interval $[0, T]$ implies that the first term of the gradient component according to the reaction coefficient $k_{m}$ vanishes. Hence, the reaction coefficient in pipe $m$ cannot be updated.

\section{References}

Al-Omari, A., Abdulla, F., 2009. A model for the determination of residential water demand by the use of tracers. Advances in Engineering Software 40, 85-94.

Alcocer-Yamanaka, V., Tzatchkov, V., Arreguin-Cortes, F., 2012. Modeling of drinking water distribution networks using stochastic demand. Water Resour Manage 26, 1779-1792.

Andrews, M., 2013. The use of dual-number-automatic-differentiation with sensitivity analysis to investigate physical models. ASME J. Fluids Eng. $135,10 \mathrm{p}$.

Bakker, M., Vreeburg, J., van Schagen, K., Rietveld, L., 2013. A fully adaptive forecasting model for short-term drinking water demand. Journal of Environmental Modelling and Software 48, 141-151. 
Becker, R., Rannacher, R., 2001. An optimal control approach to a posteriori error estimation in finite elements methods. Acta Numerica, Cambridge Press 10, 1-102.

Berry, J., Fleischer, L., Hart, W., Philips, C., Watson, J.-P., 2005. Sensor placement in municipal water networks. J. Water Resour Plann Manage $131(3), 237-243$.

Berry, J., Hart, W., Philips, C., Uber, J., Watson, J.-P., 2006. Sensor placement in municipal water networks with temporal integer programming models. J. Water Resour Plann Manage 132 (4), 218-224.

Buchberger, S., Wells, G., 1996. Intensity, duration, and frequency of residential water demands. Journal of Water Resources Planning and Management $122(1), 11-19$.

Cominola, A., Giuliani, M., Piga, D., Castelletti, A., Rizzoli, A., 2015. Benefits and challenges of using smart meters for advancing residential water demand modeling and management: a reveiw. Journal of Environmental Modelling and Software 72, 198-214.

Elbern, H., Schmidt, H., Talagrand, O., Ebel, A., 2000. 4d-variational data assimilation with an adjoint air quality model for emission analysis. Journal of Environmental Modelling and Software 15, 539-548.

Fabrie, P., Gancel, G., Mortazavi, I., Piller, O., 2010. Quality modeling of water distribution systems using sensitivity equations. J. Hydraul. Eng. $136(1), 34-44$.

Gancel, G., 2006. Modelisation d'un probleme inverse pour la qualite de 
l'eau dans les reseaux d'eau potable (in french). Ph.D. thesis, University Bordeaux I.

Jonkergouw, P., Khu, S., Kapelan, Z., Savic, D., 2008. Water quality calibration under unkonwn demands. J. Water Resour Plann Manage 134 (4), $326-336$.

Kang, D., Lansey, K., 2009. Real-time demand estimation and confidence limit analysis for water distribution systems. Journal of Hydraulic Engineering 135 (10), 825-837.

Kauker, F., Kaminski, T., Karcher, M., Dowdall, M., Brown, J., Hosseini, A., Strand, P., 2016. Model analysis of worst place scenarios for nuclear accidents in the northern marine environment. Journal of Environmental Modelling and Software 77, 13-18.

Kessler, A., Ostfeld, A., Sinai, G., 1998. Detecting accidental contaminations in municipal water networks. J. Water Resour Plann Manage 124 (4), 192198.

Krause, A., Leskovec, J., Guestrin, C., VanBriesen, J., Faloutsos, C., 2008. Efficient sensor placement optimization for securing large water distribution networks. J. Water Resour Plann Manage 134 (6), 516-526.

Lee, B., Deininger, R., 1992. Optimal locations of monitoring stations in water distribution system. J. Environ. Eng. 118 (1), 4-16.

Liggett, J., Chen, L.-C., 1994. Inverse transient analysis in pipe networks. Journal of Hydraulic Engineering 120 (8), 934-955.

Meseguer, J., Mirats-Tur, J., Cembrano, G., Puig, V., Quevedo, J., Perez, R., Sanz, G., Ibarra, D., 2014. A decision support system for on-line 
leakage localization. Journal of Environmental Modelling and Software $60,331-345$.

Munavalli, G., Kumar, M. M., 2005. Water quality parameter estimation in a distribution system under dynamics state. Water Research 39, 4287-4298.

Oden, J. T., Prudhomme, S., 2002. Estimation of modeling error in computational mechanics. J. Comput. Phys. 182, 496-515.

Pasha, M., Lansey, K., 2012. Effect of data collection on the estimation of wall reaction coefficients for water distribution models. J. Water Resour Plann Manage 138 (6), 614-623.

Powell, J., West, J., Hallam, N., Forster, C., Simms, J., 2000. Performance of various kinetic models for chlorine decay. J. Water Resour Plann Manage $126(1), 13-20$.

Preis, A., Ostfeld, A., 2008. Multiobjective contaminant sensor network design for water distribution systems. J. Water Resour Plann Manage $134(4), 366-377$.

Rodriguez, M., West, J., Powell, J., Serodes, J., 1997. Application of two approaches to model chlorine residuals in severn trent water (stw) distribution systems. Water Science and Technology 36 (5), 317-324.

Rossman, L., Boulos, P., 1996. Numerical methods for modeling water quality in distribution asystems: a comparison. J. Water Resour Plann Manage $1222(2), 137-146$.

Rossman, L. A., 2000. EPANET users' manual. U.S. Environmental Protection Agency, National Risk Management Research Laboratory, Office of Research and Development, Cincinnati. 
Sharp, W., Pfeffer, J., Morgan, M., 1991. Insitu chlorine decay rate testing. AWWA Research Foundation and USEPA, Cincinnati, Ohio, pp. 311-322.

Vasconcelos, J., Rossman, L., Grayman, W., Boulos, P., Clark, R., 1997. Kinetics of chlorine decay. Journal-American Water Works Assoc. 89 (7), $54-65$.

Waeytens, J., Chamoin, L., Ladevèze, P., 2012. Guaranteed error bounds on pointwise quantities of interest for transient viscodynamics problems. Computational Mechanics 49 (3), 291-307.

Waeytens, J., Chatellier, P., Bourquin, F., 2013. Sensitivity of inverse advection-diffusion-reaction to sensor and control: a low computational cost tool. Computers and Mathematics with Applications 6 (66), 10821103.

Waeytens, J., Chatellier, P., Bourquin, F., 2015. Inverse computational fluid dynamics: influence of discretisation and model errors on flows in water network including junctions. ASME Journal of Fluids Engineering 137 (9), $17 \mathrm{p}$.

Waeytens, J., Chatellier, P., Bourquin, F., 2017. Impacts of discretisation error, flow modeling error and measurement noise on inverse transportdiffusion-reaction in a t-junction. ASME Journal of Fluids Engineering (in press).

Xu, J., Fischbeck, P., Small, M., VanBriesen, J., Casman, E., 2008. Identifying sets of key nodes for placing sensors in dynamic water distribution networks. J. Water Resour Plann Manage 134 (4), 378-385. 